\title{
A fast-reciprocating probe array for two-dimensional measurements in the divertor region of the Tokamak à Configuration Variable
}

\author{
H. De Oliveira, ${ }^{1}$, a) C. Theiler, ${ }^{1}$ H. Elaian, ${ }^{1}$ and the TCV team ${ }^{1,}$ b) \\ Swiss Plasma Center (SPC), École Polytechnique Fédérale de Lausanne (EPFL), CH-1015 Lausanne, \\ Switzerland
}

(Dated: 19 March 2021)

A detailed description of the construction and commissioning of the fast-moving Reciprocating Divertor Langmuir Probe Array (RDPA) on the Tokamak à Configuration Variable (TCV) is presented. A horizontal array of 24 Langmuir probes (12 Mach probes) combined with a fast vertical movement provides unprecedented two-dimensional measurements of the plasma properties across the entire divertor outer leg volume and up to the X-point. The L-shape probe arm has to be very compact to minimize plasma perturbations and, at the same time, has to house 24 coaxial transmission lines, withstand time averaged heat fluxes of up to $\approx 30 \mathrm{MW} / \mathrm{m}^{2}$, accelerations of up to $8 \mathrm{~g}$, and be resilient to violent plasma disruptions. For the fast vertical motion of the probe arm, extending up to $38 \mathrm{~cm}$ into the TCV vacuum vessel, an assembly with a precise and powerful linear electric motor (up to $4000 \mathrm{~N}$ of force) was mounted in a $4 \mathrm{~m}$ high structure within the limited space below TCV. The diagnostic has already performed measurements in $\approx 200$ plasma discharges, and has been hit by $\approx 20$ disruptions without suffering any critical damage. The measurements are qualitatively and quantitatively consistent with other reference diagnostics, such as upstream electron Thomson scattering and downstream wall-embedded Langmuir probes and are used extensively on TCV for experimental divertor studies and for comparisons with edge transport and turbulence code results.

\section{Introduction}

In future tokamaks, such as ITER and DEMO, wall materials will be subjected to considerably higher heat fluxes than in present-day machines. Maintaining plasma facing components in good condition and simultaneously assuring acceptable core performance will be an outstanding challenge. The situation is further complicated by the fact that extrapolations towards future tokamaks is difficult and the mechanisms governing transport of energy and particles are still being investigated. Detailed diagnostic coverage of the boundary plasma of currently operational machines is therefore crucial. In the Tokamak à Configuration Variable $(\mathrm{TCV})^{1}$, Langmuir probes 2 (LPs) are one of the most powerful diagnostics to describe local properties in the boundary region of the plasma 3 4. Wall-embedded LPs have been installed for more than 20 years in TCV 5 with an extended poloidal coverage. LPs are also present at the outboard midplane, mounted on a fast reciprocating probe 6 7 undergoing a scanning radial motion. The region in between the upstream plasma and the plasma at the target is diagnosed with other systems such as multispectral imaging ${ }^{8}$, divertor electron Thomson scattering 9 and spectroscopic measurements 10 . However, these measurements differ in many aspects from the quantities obtained from LPs and can be subjected to limited spatial resolution and/or coverage (Thomson scattering, divertor spectroscopy) and time resolution (Thomson scattering, divertor spectroscopy, multispectral imaging). In particular, a coverage of the divertor volume with Langmuir

\footnotetext{
a) hugo.deoliveira@epfl.ch

b) See author list of S. Coda et al 2019 Nucl. Fusion 59112023
}

probes allows to resolve the turbulence time scales and to measure quantities which are difficult to deduce from spectroscopy, such as parallel flows and plasma potential.

The divertor and $\mathrm{X}$-point regions of tokamaks have been previously diagnosed with fast reciprocating Langmuir probes, such as in DIII-D ${ }^{11}$, Asdex Upgrade ${ }^{[2 \mid 13}$ and JT-60U14. The DIII-D probe comes closest to providing $2 D$ measurements in the tokamak divertor. Indeed, sweeping of the magnetic equilibrium between repeat discharges combined with several reciprocations have been used to reconstruct 'reduced' $2 D$ maps ${ }^{15 / 16}$. Large Langmuir probe arrays have also been used on various fusion devices, although usually not mounted on a fast reciprocating arm. For example, in the Caltech tokamak, $1 D$ and $2 D$ arrays have been mounted on limiters at the outer-mid plane ${ }^{17}$ and enabled one of the earliest studies performed to describe the plasma turbulence properties in the Scrape-Off-Layer (SOL). Another example is the stellarator Wendelstein 7-X, where, in the divertor region, several LP arrays have been mounted on a large reciprocating manipulator $\frac{18}{}$, aimed primarily to expose samples to reactor relevant target plasma conditions.

In this paper, we present the Reciprocating Divertor Probe Array (RDPA) on TCV, which, thanks to the open vessel geometry of TCV, enables detailed 2D Langmuir probe measurements across an extended part of the divertor region. This is achieved by combining the fast vertical motion of a linear motor (up to $38 \mathrm{~cm}$ into the chamber, at speed of up to $\left.2.6 \mathrm{~ms}^{-1}\right)$ and a radial array of 12 rooftop Mach probes (1 $\mathrm{cm}$ radial resolution). In the following, the main design constraints of this new diagnostic are discussed in SEC. II. followed by a detailed presentation of the probe design in SEC. III. First example measurement results are presented in SEC. IV. followed by the conclusions. 


\section{Main design constraints}

\section{A. Probe tip requirements for $J_{s a t}$ and Mach number measurements}

In a strongly magnetized plasma, the effective ion collection area of the probe is computed as the projection of the probe surface in the direction of the magnetic field 4. This approach is justified if the ion Larmor radius and the Debye length are smaller than the probe dimensions across the magnetic field. The typical ion temperature in the TCV SOL is expected to be $T_{i} \approx 15 \mathrm{eV}$, the TCV magnetic field is $\approx 1.5 \mathrm{~T}$ and we consider deuterium ions. The corresponding Larmor radius is $\rho_{\text {Larmor }}=\frac{m_{i}\left\langle v_{\perp}\right\rangle}{e B}=\frac{\sqrt{\pi m_{i} e T_{i}}}{\sqrt{2} e B} \approx 0.43 \mathrm{~mm}$ where $\left\langle v_{\perp}\right\rangle$ is taken as the average norm of the velocity in a $2 D$ Maxwellian distribution, $m_{i}$ is the deuterium ion mass, $e$ is the elementary charge, $T_{i}$ is the ion temperature (expressed in $\mathrm{eV}$ ) and $B$ is the norm of the magnetic field. The Debye sheath width (equal to a few times the Debye length ${ }^{3}$ ) is much smaller than $\rho_{\text {Larmor }}$ for typical TCV plasmas: $\lambda_{\text {Debye }}=\sqrt{\frac{\epsilon_{0}\left(T_{e}+T_{i}\right) e}{n_{e} e^{2}}} \approx 0.013 \mathrm{~mm}$. Probe tips heights of $1 \mathrm{~mm}>2 \rho_{\text {Larmor }}$ have been chosen for RDPA. This $1 \mathrm{~mm}$ dimension is also used for dome shaped LPs in TCV $\sqrt{519}$ and yields more consistent results than flush LPs for various reasons (reduced sheath expansion, less relative error on the height and better signal for angles between the wall surface and the magnetic field lines $\alpha \approx 0^{\circ}$ ). Probe tips must be split in two to allow for measurements of parallel Mach numbers 4 , separating them in an upstream and a downstream part. The Gundestrup Mach probe design 20, 21 would allow to obtain more information, such as perpendicular flows, but it has been discarded because it adds complexity to build an array of tightly packed probes.

\section{B. Spatial coverage requirements}

Several probe motions are possible such as rotation 22 , horizontal 12 and vertical translation 11. A rotational system can hardly rest within the limited shadow of the TCV tiles, protruding only $20 \mathrm{~mm}$ above the vacuum vessel floor. If the rotational system is installed in the largest port diameter ( $\approx 10 \mathrm{~cm}$ radius $)$, it would be impossible to probe the $\mathrm{X}$-point region. In the case of horizontal motions, the disadvantage is that the region near the target can not be covered because the lowest lateral ports axis are located $38 \mathrm{~cm}$ above the floor. Due to these limitations for the rotary and horizontal motions, a vertical motion was considered optimal. A radial array of probe tips mounted on a horizontal arm was found to be the best choice. This arm is held in place by a vertical shaft. The necessary radial dimension of this array is determined from experimental results on relevant TCV plasmas: the upstream e-folding lengths of the heat flux $\lambda_{q}$, the electron temperature $\lambda_{T_{e}}$ and the density $\lambda_{n}$ are usually found in the $2-20 \mathrm{~mm}$ range $23 \mid 24$. This width increases in the divertor due to the geometry of the magnetic field (the poloidal flux expansion $f_{x}$ can vary by factors of $\left.2-20^{(25}\right)$. Let us consider an intermediate case:

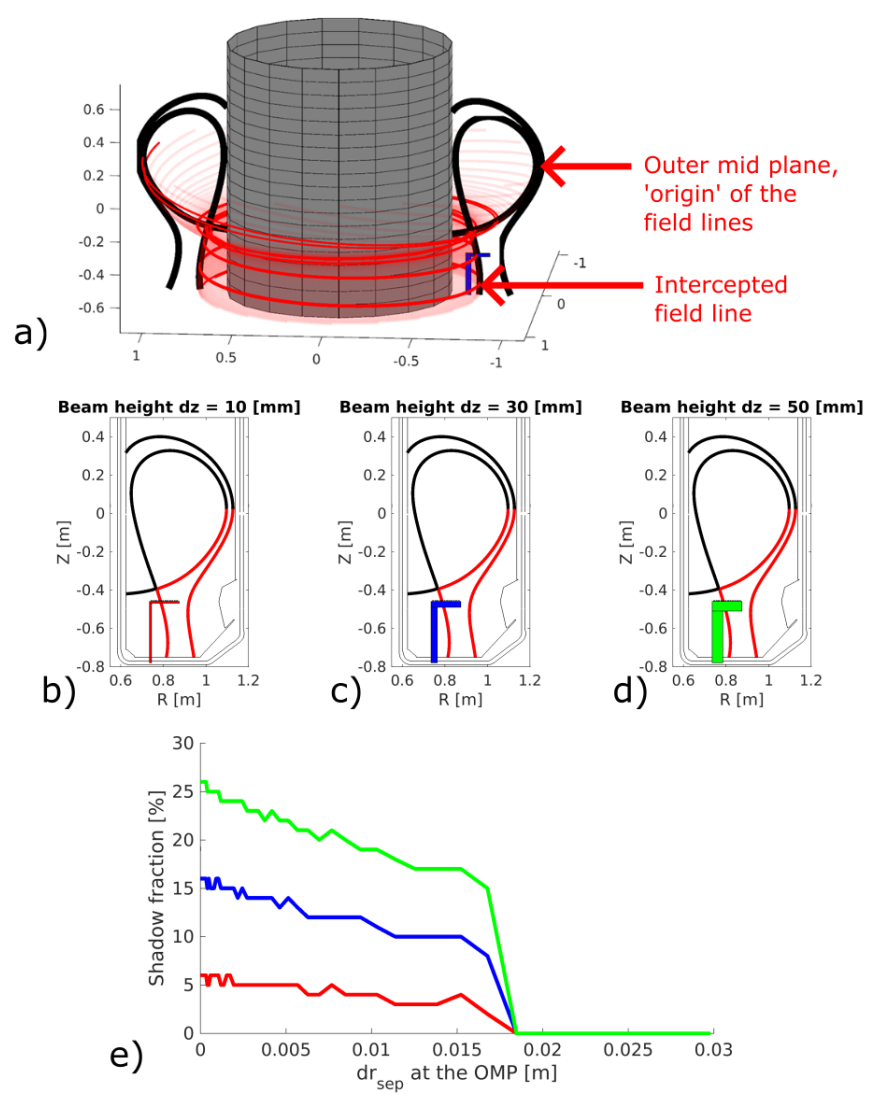

FIG. 1. a) Magnetic field lines in the SOL from the mid plane to the outer target with the highlighted magnetic field line intercepted by RDPA, b), c) and d) illustrations of different probe arm sizes (respectively $10 \mathrm{~mm}, 30 \mathrm{~mm}$ and $50 \mathrm{~mm}$ ) and the magnetic equilibrium chosen for the calculations and e) calculated fractions of magnetic field lines being intercepted by the different probe arm sizes.

$\lambda_{u}=10 \mathrm{~mm}$ and $f_{x}=10$. If the outer divertor leg is vertical, the RDPA radial coverage should be larger than the SOL width at the target: $\lambda_{\text {target }} \approx \lambda_{u} * f_{x}=100 \mathrm{~mm}$. This criterion is fulfilled by a $13 \mathrm{~cm}$ radial extension of the RDPA probe, with the probe tips covering a radial range of $11 \mathrm{~cm}$. The effective spatial resolution of RDPA, i.e. relative to the upstream profile, is a function of the flux expansion in the divertor. The 12 tips in the RDPA array give a $10 \mathrm{~mm}$ radial resolution. This corresponds to an 'upstream mapped' radial resolution of $1 \mathrm{~mm}$ for a poloidal flux expansion of $f_{x}=10$. Strike point sweeping between a first and a second plunge (or between upwards and downwards strokes) may be performed to improve the resolution for specific studies.

\section{Minimizing plasma perturbations: probe shadow and recycling}

Heat and particles are expelled from the core plasma near the outer mid plane and flow along the magnetic field to the wall, see FIG. 1 a). This flow can be intercepted by 

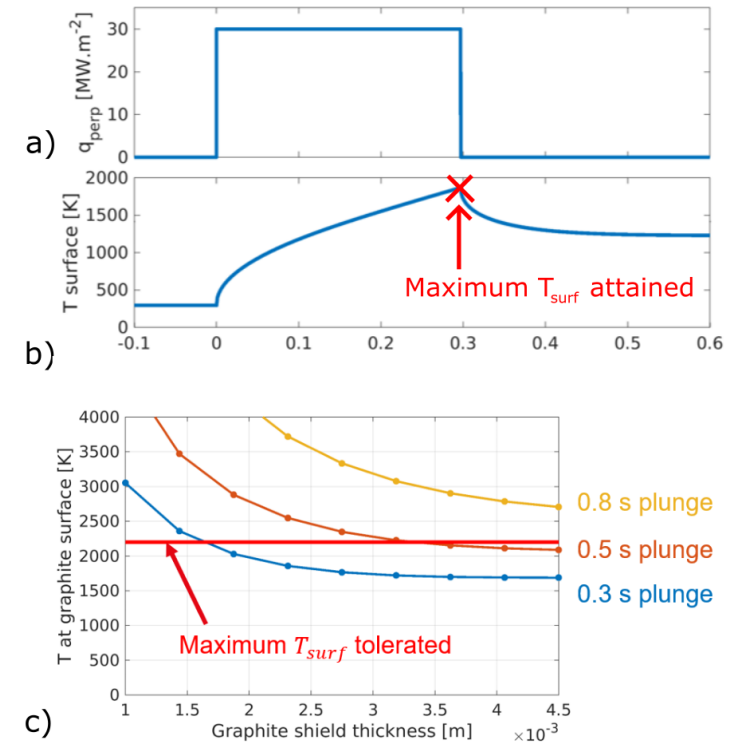

FIG. 2. Output from the $1 D$ model for the heat diffusion within the graphite shield at a given heat flux $q_{\|}=$ $30 M W \cdot m^{-2}$, heat capacity $c_{p}=1.72 \mathrm{~kJ} \mathrm{~kg}^{-1} \mathrm{~K}^{-1}$ and heat conductivity $\lambda=47 \mathrm{Wm}^{-1} \mathrm{~K}^{-1}$. a) Applied heat flux as a function of time, $\mathbf{b}$ ) temperature at the graphite shield surface for a given shield thickness $d=3 \mathrm{~mm}$, and a plunge duration $t=0.3 \mathrm{~s}$ and c) maximum surface temperatures gathered from several simulations with different graphite shield thicknesses and different plunge times.

the probe and thus strongly perturbing the local plasma. In order to limit the perturbation and the fraction of intercepted field lines, $f_{\text {shadow }}$, an inverted L-shape has been chosen for the in-vessel structure, see FIG. 1 b), c) and $\mathbf{d}$ ): the vertical pole remains in the private plasma (or in the far SOL if the probe is rotated and the leg is moved to a smaller radius) and the compact horizontal beam hosts the radially distributed probe tips. $f_{\text {shadow }}$ is calculated for each flux surface. A sufficiently large number of equally distributed field lines are required, because field line tracing is here performed as a discrete process: $f_{\text {shadow }}=N_{\text {intercepted }} / N_{\text {total }}$, where $N_{\text {intercepted }}$ is the number of field lines intercepted by the probe and $N_{\text {total }}$ is the total number of field lines initially generated for a given flux surface. $f_{\text {shadow }}$ increases linearly with the vertical extent of the horizontal beam, see FIG. 1] e). $15 \%$ shadowing, considered to be an acceptable fraction, requires a compact probe beam of not more than $\approx 3 \mathrm{~cm}$ in height.

\section{Minimizing impurity release and material erosion}

Graphite grade SGL ${ }^{\circledR}$ R6650 is the preferred choice for plasma facing components at TCV because the entire wall is currently covered with tiles of the same material 26. This material shows excellent thermal shock resistance and can be easily machined. The maximum service temperature is considered to be the onset of radia- tion enhanced thermal sublimation at $T_{\text {surf }} \approx 2200 K^{27}$. In order to determine the adequate graphite heat shield thickness and the acceptable exposure times, $1 D$ thermal analysis have been performed in this study. In order to simplify calculations 26 , the graphite properties have been set to be constant as a function of temperature by calculating the average quantities in the relevant temperature range $293 K<T<2000 K$ : the thermal conductivity is $\lambda_{\text {average }}=47 \mathrm{~W} /(\mathrm{m} . \mathrm{K})$ and the heat capacity is $c_{\text {average }}=1720 \mathrm{~J} /(\mathrm{kg} . \mathrm{K})$. In order to estimate the heat flux onto the probe body, the worst plasma conditions were chosen: $T_{e}=T_{i}=50 \mathrm{eV}$ and $n_{e} \approx 1.5 \cdot 10^{19} \mathrm{~m}^{-3}$ 28. The transmitted power through the sheath, parallel to the magnetic field, is approximated with the formula 3: $q_{\|}=\gamma \cdot e \cdot T_{e} \cdot \Gamma \approx 25 \mathrm{MWm}^{-2}$, assuming $T_{i}=T_{e}$ and $\gamma=6[29$, and with $\Gamma$ the particle flux density, defined as $\Gamma=\frac{1}{2} n_{e} \cdot \sqrt{\frac{e \cdot\left(T_{i}+T_{e}\right)}{m_{i}}}$. The heat flux chosen for the calculations has been set above this result with some margin: $q_{\|}=30 \mathrm{MWm}^{-2}$. The results from a single simulation of the $1 D$ model are shown as an example in the case of a $3 \mathrm{~mm}$ thick shield, $0.3 \mathrm{~s}$ plunge duration and thus a heat flux perpendicular to the graphite shield of $30 \mathrm{MW} \cdot \mathrm{m}^{-2}$, see FIG 2 a) and b). In this example, the peak surface temperature of the graphite heat shield is $\approx 1730 \mathrm{~K}$. The calculations have been then performed for various shield thicknesses and various exposure times, see FIG. 2 c). As expected in case of inertial cooling, increasing the shield thickness is not sufficient to remain below the surface temperature limit. After a certain thickness, the reduction in surface temperature with increasing thickness is negligible, see the yellow curve corresponding to the long plunge time $\delta t=0.8 s$ in FIG. 2 $\mathbf{c}$ ). A relatively short exposure time and therefore a fast probe motion, shorter than $\delta t=0.5 \mathrm{~s}$, is thus mandatory. The best compromise is found at $\delta t=0.4 s$ and shield thickness $2.5 \mathrm{~mm}$. In order to reciprocate within the $0.4 \mathrm{~s}$ allocated along the $0.38 \mathrm{~m}$ distance from the floor to the $\mathrm{X}$-point, a high acceleration, $a=80 \mathrm{~m} \cdot \mathrm{s}^{-2}$, and a high peak velocity, $v=2.5 \mathrm{~m} . \mathrm{s}^{-1}$, are necessary.

\section{E. Ensuring probe integrity during off normal events}

Off normal plasma events, such as disruptions, threaten the probe integrity. Disruptions are frequent in TCV experiments, so RDPA must be able to withstand a large number of them. In typical disruptions, the sudden loss of energetic particle confinement, the thermal quench, is followed by a current quench due to the drop in plasma conductivity 30. The current quench is the most hazardous due to the large halo currents which dissipate some of the magnetic energy stored in the plasma. Once the plasma becomes more resistive than the vacuum vessel, a substantial fraction of the current could be transferred from the plasma to the vessel through the probe arm. In the case where $5 \%$ of the overall plasma current in a typical experiment $I_{p}=320 \mathrm{kA}$ would flow vertically in the RDPA structure, the corresponding Lorentz force would be $\vec{F}=\vec{I} \times \vec{B} L=(16 k A \cdot 1.5 T \cdot 0.35 \mathrm{~m}) \cdot \pm \overrightarrow{e_{r}} \approx$ $8400 N \cdot \pm \overrightarrow{e_{r}}$. Such a large transversal force would dam- 
age the structure and therefore the graphite and metallic structure must be electrically insulated from each other. In response to this threat, several layers of electrical insulations have been installed in RDPA, see SEC. III for more details.

\section{F. Various external constraints}

The baking temperature of the TCV vacuum vessel is $\approx 220{ }^{\circ} \mathrm{C}$. Tin, aluminum and polymers are forbidden due to the potential creep and out-gassing at this temperature. Creep in polymers is particularly worrying in the case of electrical insulation washers because the applied torque in the screw can be lost during baking. All electrical insulation washers must be made out of creep free material such as ceramic materials (Muscovite mica sheet, aluminum nitride).

The magnetic field in the toroidal coils is usually higher than $1 \mathrm{~T}$, thus preventing the installation of any magnetic component such as an electrical motor close to the plasma. Under the toroidal coils, the highest magnetic field is generated by the central solenoid and its direction is nearly vertical. The vertical magnetic field is low enough to permit the use of magnetic components: $\approx \pm 0.05 \mathrm{~T}$ in the beginning of the discharge and is ramped up/down at $\approx \mp 0.05 \mathrm{~T} / \mathrm{s}$.

The space available for RDPA is constrained by the port availability in TCV. Fortunately, there is less competition for the ports under the machine because access is more difficult. At the sector 04 , the vacuum port is centred at $R=0.8 \mathrm{~m}$ and has a diameter $d=150 \mathrm{~mm}$, therefore sufficiently large to satisfy the radial coverage requirement presented in SEC. II B. The vertical motion system must fit in the limited space between the toroidal coils, $\approx 0.7 \mathrm{~m}$ below the vacuum vessel.

\section{Probe design}

Before presenting the RDPA probe design in detail in this section, we show in FIG. 3 a brief overview. Subplots a), b), and c) show the probe during assembly, once installed inside TCV and fully deployed, and when in rest position, respectively. The dark grey parts of RDPA, with the same colour as the rest of the TCV walls in FIG. 3 b), are graphite heat shields. The white parts are made out an electrical insulator refractory material, Boron Nitride $(\mathrm{BN})$. The diagnostic structure, $\approx 4 m$ high, is mounted on the TCV basement floor, see FIG. 3 d). FIG. 3 e) shows a specially filtered snapshot $(D-\beta$ line from the MANTIS imaging system ${ }^{8}$ ) of the plasma at a time when RDPA is close to the maximum vertical position. Some plasma-probe interaction is clearly visible, as well as a characteristic shadow along the magnetic field. Finally, FIG. 3 f) shows an example of the $2 D$ measurements achieved with the RDPA, in this case time-averaged profiles of the ion saturation current density $\left(J_{\text {sat }}\right)$ recorded by the upstream probe tips. Values and discussion of the first measurements are given later, in SEC. IV
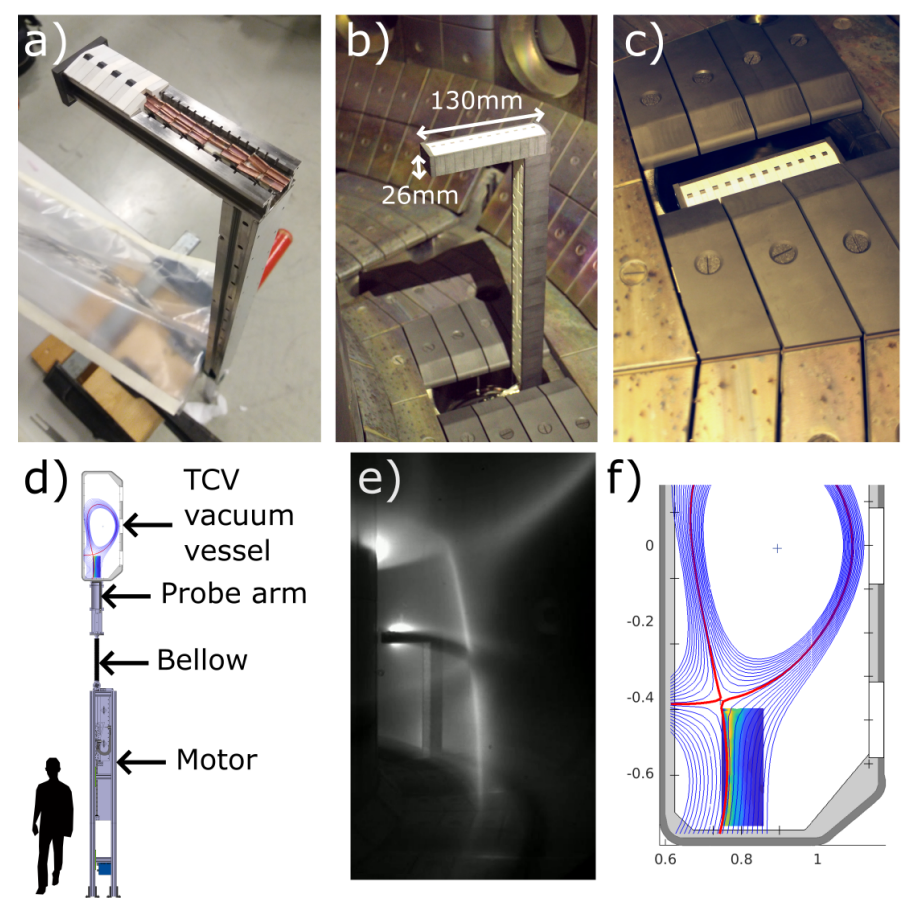

FIG. 3. a) RDPA during assembly, b) RDPA inside the vacuum vessel at maximum height, c) RDPA inside the vacuum vessel in rest position, $\mathbf{d}$ ) poloidal view of the entire diagnostic mounted in the TCV environment, e) $D-\beta$ light snapshot recorded with the MANTIS camera system 8 of the RDPA plunge in a lower single null plasma, discharge \#63023, f) example of a measured profile: ion saturation current density from the upstream RDPA probe tips, discharge \#63023.

\section{A. Probe head design}

The probe head of RDPA is made of a titanium hollow structure in order to provide the highest stiffness to weight ratio possible. The structure enables to host the 24 transmission lines. If not in use, the probe head rests $\approx 2 \mathrm{~cm}$ below the upper surface of the carbon divertor in order to avoid unnecessary damage from experiments unrelated to the diagnostic. The $38 \mathrm{~cm}$ plunge is actually only $36 \mathrm{~cm}$ in the plasma, just enough to reach the X-point for diverted plasma at $Z=0 \mathrm{~m}$. The horizontal beam height is $26 \mathrm{~mm}$, see FIG. 3 b). Further reduction in the beam height is believed to be impossible while maintaining the same number of cables with the current design. The only surfaces in contact with the plasma are the probe tips, the graphite and the BN thermal shields. The thermal shields are slid radially in place during the probe assembly, see FIG. 4 b) for an intermediate state of the assembly. The tight tolerances and the friction in between parts is sufficient to avoid any jiggling motion. Probe tips are pushed upward by canted niquel alloy springs resting on copper parts, see FIG. 4 a). The spring provides electrical contact to the transmission line conductors and ensures that the probe tip position relative to the $\mathrm{BN}$ shield does not change. 


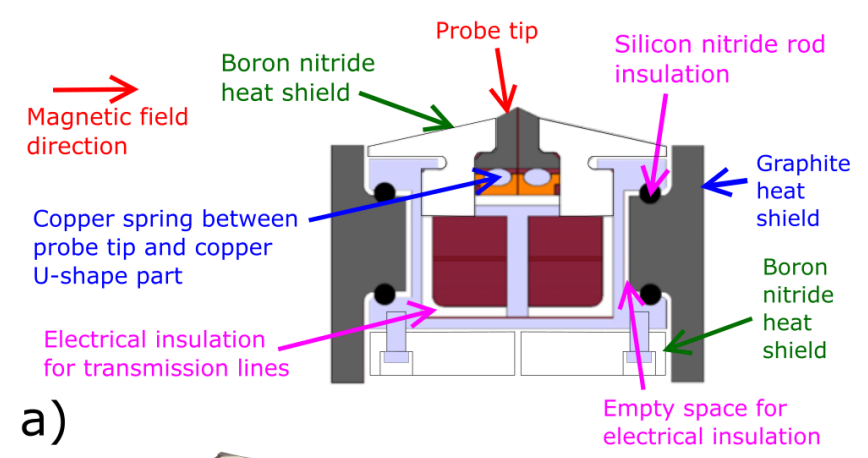

a)

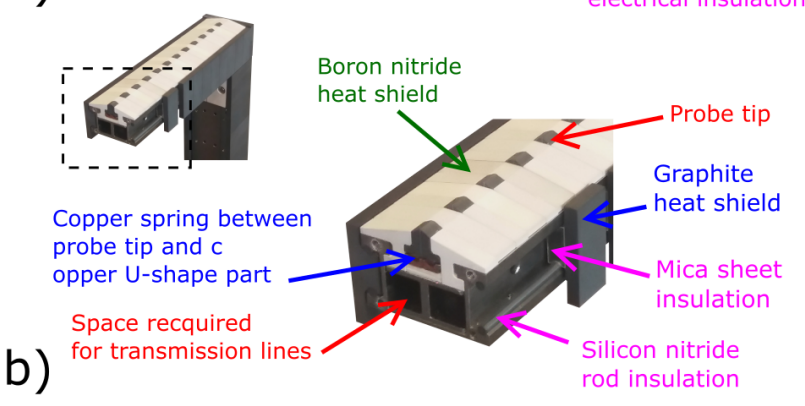

a)

b)

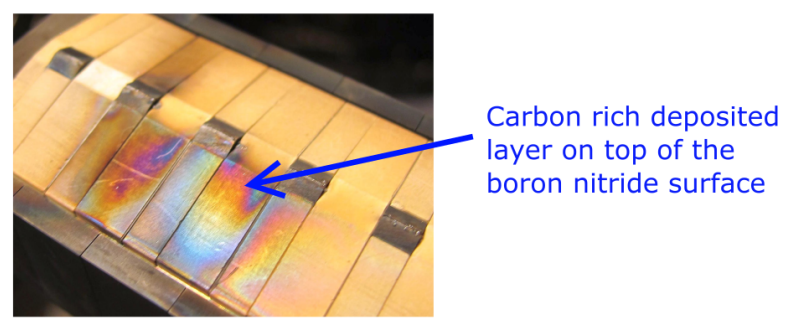

c)

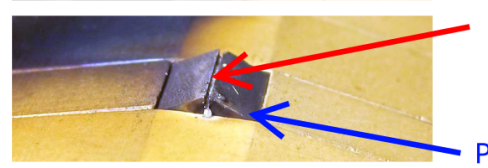

Mica sheet edge between Mach probes

Probe tip with sharp edge:

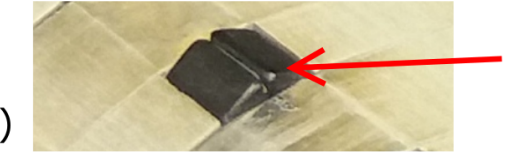

Probe tip with smooth edges to avoid arcing

FIG. 5. a) Picture of the RDPA probe tips after exposure to the plasma near the separatrix with the diffraction pattern caused by the carbon co-deposit, $\mathbf{b}$ ) magnified picture of the Mach tips and the mica sheets and c) probe tips modified (edges have been rounded up to avoid arcing) ready for the next experimental campaign.

conductive) are insulated from the L-shaped structure. The parts are mechanically linked through ceramic rods, see FIG. 4 a. The $\approx 1 \mathrm{~mm}$ gap between the structure and the shield can fail to electrical insulate if an electrical arc can develop. However, the resistivity of the electrical arc is sufficiently high to limit the highest current excursions. 5) All the graphite thermal shields (10 $\mathrm{mm}$ wide) are insulated from each other with mica sheet insulations, see $4 \mathrm{~b}$ ). The shield voltages are therefore following the local floating potentials dictated by the plasma sheath condition.

The probe tips protrude $1 \mathrm{~mm}$ above the BN shields,

FIG. 4. a) Drawing of the probe head cross section with labelled parts, $\mathbf{b}$ ) picture of the probe head during the assembly and c) cross sectional view of the L-shaped structure with the different parts labelled depending on their voltage reference.

In order to ensure proper protection from the halo currents discussed in SEC. II E, 5 different protections have been implemented. 1) The metallic support structure of RDPA (connected to the tokamak ground voltage) is always maintained below the level of the floor, even for the maximum plunge height. 2) The L-shaped titanium structure is disconnected from the rest of the diagnostic with electrically insulated screws, see FIG. 4 c). 3) The transmission lines must be insulated from the structure in both the vertical and horizontal parts of the L-shape structure, see FIG. 4 a). Indeed, the transmission lines outer jacket are connected to the tokamak ground in order to reduce capacitive and inductive effects for the measurements. 4) The graphite thermal shield (electrically see FIG. 5. A thin Muscovite mica sheet is inserted to insulate the upstream from the downstream Mach probe tips, see FIG. 5 b). The probe tips have the so-called 'rooftop' shape with a reasonably low angle between the magnetic field and the surface in order to reduce the perpendicular heat flux. A significant heat sink volume is located below the surface, as shown in FIG. 4 a). The heat sink part is longer $(10 \mathrm{~mm})$ than the probe tips $(4 \mathrm{~mm})$ in the radial direction in order to increase the number of electrical contact points with the canted niquel alloy spring located below the probe tip. A significant issue that appeared during the first experimental campaign is tive voltage is applied to the probe tips, a large transient current corresponding to electrons exiting the surface is collected instead of the standard incoming ion saturation current. Smoothing the probe tips edges with sandpaper, see FIG. 5 c), has solved the problem, possibly by reducing the electrical field and the surface temperature at the tip of the conductor, both parameters being important the phenomenon of probe tips "arcing": when a nega- 
for electron emission 31 .

The lateral graphite heat shields are $2.5 \mathrm{~mm}$ thick, in accordance with the result from SEC. II A thermomechanical analysis for the final design of both lateral and extremities shields with the software ANSYS ${ }^{\circledR}$ was performed. The exposure time $\Delta t=0.3 \mathrm{~s}$ and the perpendicular heat flux input of $30 \mathrm{MW} \cdot \mathrm{m}^{-2}$ were chosen as previously assumed in SEC. $I I$. The resulting surface temperature $T_{\max } \approx 1440 \mathrm{~K}$ was below the $2200 \mathrm{~K}$ limit, the maximum tensile stress, $\sigma_{\text {tension }} \approx 15 \mathrm{MPa}$, below the $30 \mathrm{MPa}$ limit and the maximum compressive stress, $\sigma_{\text {compression }} \approx 84 \mathrm{MPa}$, below the $150 \mathrm{MPa}$ limit.

The BN thermal shield surfaces have been designed to intercept field lines at a shallow angle in order to reduce the peak perpendicular heat flux from $q_{\text {para }} \approx$ $30 M W \cdot m^{-2}$ down to $q_{\text {perp }} \approx q_{\text {para }} \cdot \sin \theta_{\text {tot }} \approx$ $12.2 M W \cdot m^{-2}$, where $\theta_{\text {tot }}=\theta_{B}+\theta_{\text {surf }} \approx 24^{\circ}$ is the highest angle possible between the inclined surface and the divertor magnetic field line. It was initially believed that the deuterium ion flux from the plasma could have lead to premature erosion of the BN parts and lead to plasma contamination. However it was found experimentally that erosion of the BN was absent and instead a deposited layer of carbon was found on the BN surface, as shown in FIG. 5 a) and b). The absence of BN erosion could be explained by the high stability of BN against chemical erosion by deuterium ions due to the strong chemical bond between boron and nitrogen atoms 32 .

\section{B. Out-of-vessel mechanical structure and vacuum assembly}

The outer structure is made of vertical aluminum profiles and is bolted to the TCV concrete ground floor, almost $4.5 \mathrm{~m}$ below the vacuum vessel, see FIG. $3 \mathrm{~d}$ ). The structure is covered with transparent plastic panels to avoid any accident. An additional safety system is a $12 \mathrm{~V}$ line that disconnects the motor electrical power supply if a single plastic panel is removed. The structure supports a thick aluminum plate on which the motor and guidance systems are mounted. 2 THK $^{\circledR}$ linear bearings $H S R 20$ $R$ ensure the guidance of the vertical motion. An electrical brake Zimmer ${ }^{\circledR}$ LCE2001AS1-01 clamps the rail of the linear bearings when the motor controller is turned off. The force on the brake is usually less than $40 \mathrm{~N}$ because the weight of the moving assembly is almost entirely offset by the atmospheric pressure subjected to the bellow. Guidance of the motion inside the vacuum is assured by $P E E K$ bearings $\approx 30 \mathrm{~cm}$ apart from each other, see FIG. 6 a) and b). Radial misalignment of up to $\approx 3 \mathrm{~mm}$ are tolerated between the vacuum vessel and the RDPA structure thanks to the flexibility of the RDPA shaft. Fast motions are not recommended when the vessel is still warm due to thermal expansion leading to radial misalignment and increased friction on the bearings. It was not possible to install a vacuum gate valve due to the space limitation from the toroidal coils. Instead the diagnostic vacuum relies on the tokamak turbo pumps (as for the glow antennas), see FIG.

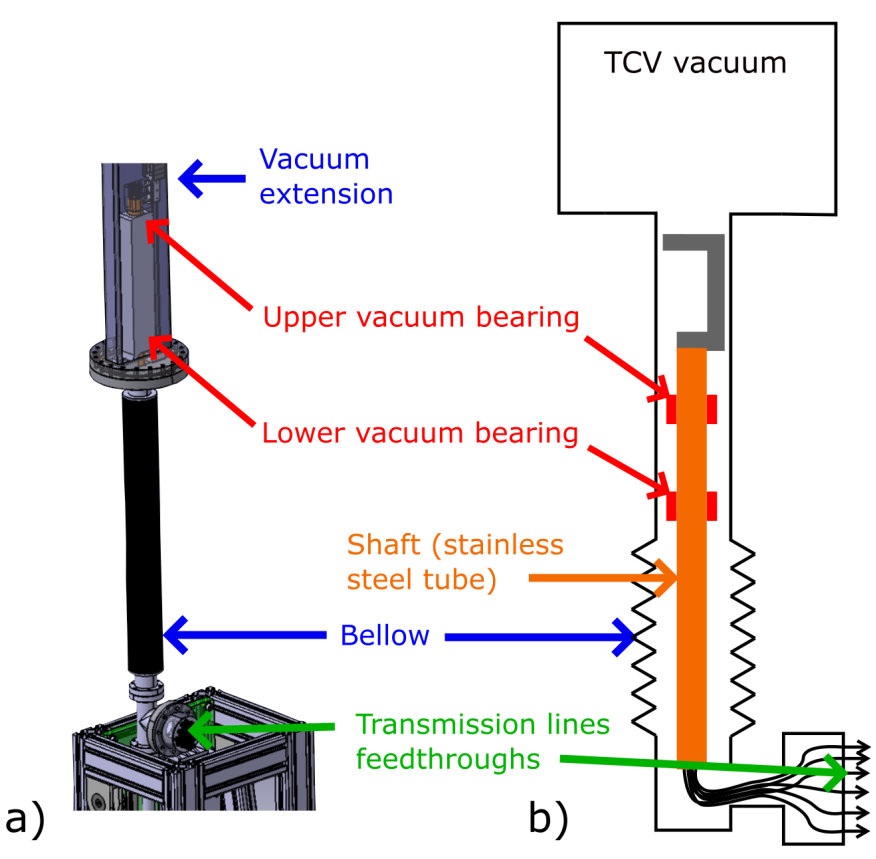

FIG. 6. a) Close up of the RDPA parts near the bellow and b) simplified sketch of the vacuum assembly cross-sectional view.

6 b). The feedthroughs for the 24 transmission lines are ceramic insulated $S M A$ connectors, welded to a custom designed vacuum flange. Frequent vacuum electrical discharge (between the conductors of the transmission lines and the tokamak ground inside the $S M A$ connectors) were observed during the first experimental campaign. The problem was later solved with additional electrical insulation jackets around the $S M A$ conductor.

\section{Motion system}

The choice of a linear electrical motor with permanent magnets was motivated by the recent success of a similar system on the Alcator C-Mod tokamak 33. Linear motors have several advantages over pneumatic systems: better position control, more flexibility in the choice of the trajectory and better reproducibility in between identical plunge motion. The position is monitored in real time by the motor controller using inputs from a Renishaw ${ }^{\mathbb{R}}$ optical encoder with sub-mm accuracy. The Aerotech $^{\circledR}$ BLMX 502 motor was chosen for RDPA because of the large trust $4400 N$ developed in the relevant speed range considered $2-3 \mathrm{~ms}^{-1}$. The sum of all the masses of the moving parts of the RDPA diagnostic is $\approx 20 \mathrm{~kg}$ and the maximum theoretical acceleration $a=\frac{F}{m} \approx \frac{4400 \mathrm{~N}}{19.4 \mathrm{~kg}}=227 \mathrm{~m} . \mathrm{s}^{-2}$ is therefore sufficient for the reference motion $\left(L=0.38 \mathrm{~m}, a=80 \mathrm{~ms}^{-2}\right.$ and $\left.v=2.5 \mathrm{~ms}^{-1}\right)$. At the time of writing, the maximum acceleration is not limited by the motor specifications but rather by the electrical connection to the power supply which could be improved. 


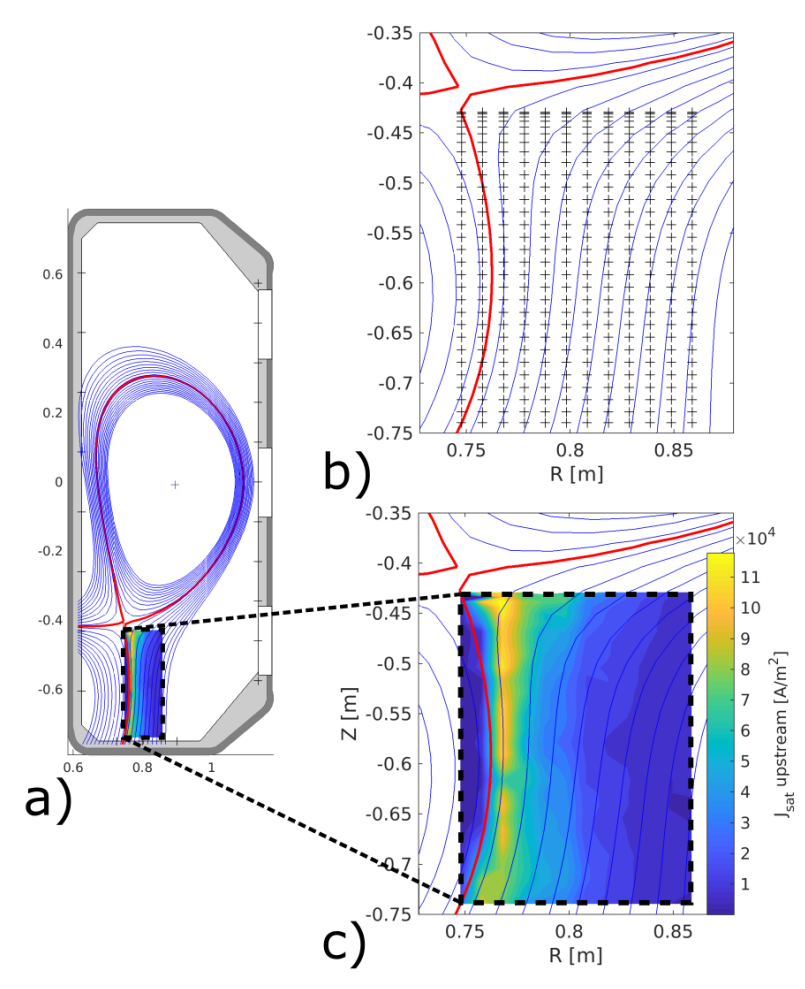

FIG. 7. a) Poloidal plane view of the TCV wall, vacuum vessel and the plasma magnetic equilibrium. b) Focus on the RDPA coverage. A contour plot of $J_{\text {sat }}$ is given here as an example in a) and $\mathbf{c}$ ).

\section{Transmission lines}

The transmission lines consist of $\approx 8 m$ of standard coaxial cables outside the machine and $\approx 2 \mathrm{~m}$ of Thermocoax ${ }^{\mathrm{R}}$ cables inside the machine (vacuum side). The Thermocoax ${ }^{\circledR}$ cables are kept within the titanium structure of the probe arm, well insulated from the plasma heat flux and are expected to remain close to room temperature. The assembly process is tedious and the transmission line would benefit from a redesign for a future version of the diagnostic. The overall DC resistance, inductance and capacitance of the transmission lines are $R \approx 3.6 \Omega, L \approx 10.9 \mu H$ and $C \approx 1.9 n F$. The large $\mathrm{DC}$ resistance $R \approx 3.6 \Omega$ (compared to $\approx 1.4 \Omega$ for wall embedded probes ${ }^{5}$ ) is problematic in the case of low electron temperature measurements 19 . The most straightforward improvements to reduce the resistance would be to use thicker coaxial cable for the line outside of the machine and to improve the contact resistance at the level of the niquel alloy canted spring. The transmission lines end at the electronics cubicles where the $2 \mathrm{MHz}$ acquisitions and the custom built amplifiers are located 5 .

\section{Example results}

In the following, first $2 D$ plots of time averaged plasma quantities obtained with RDPA in the poloidal plane are

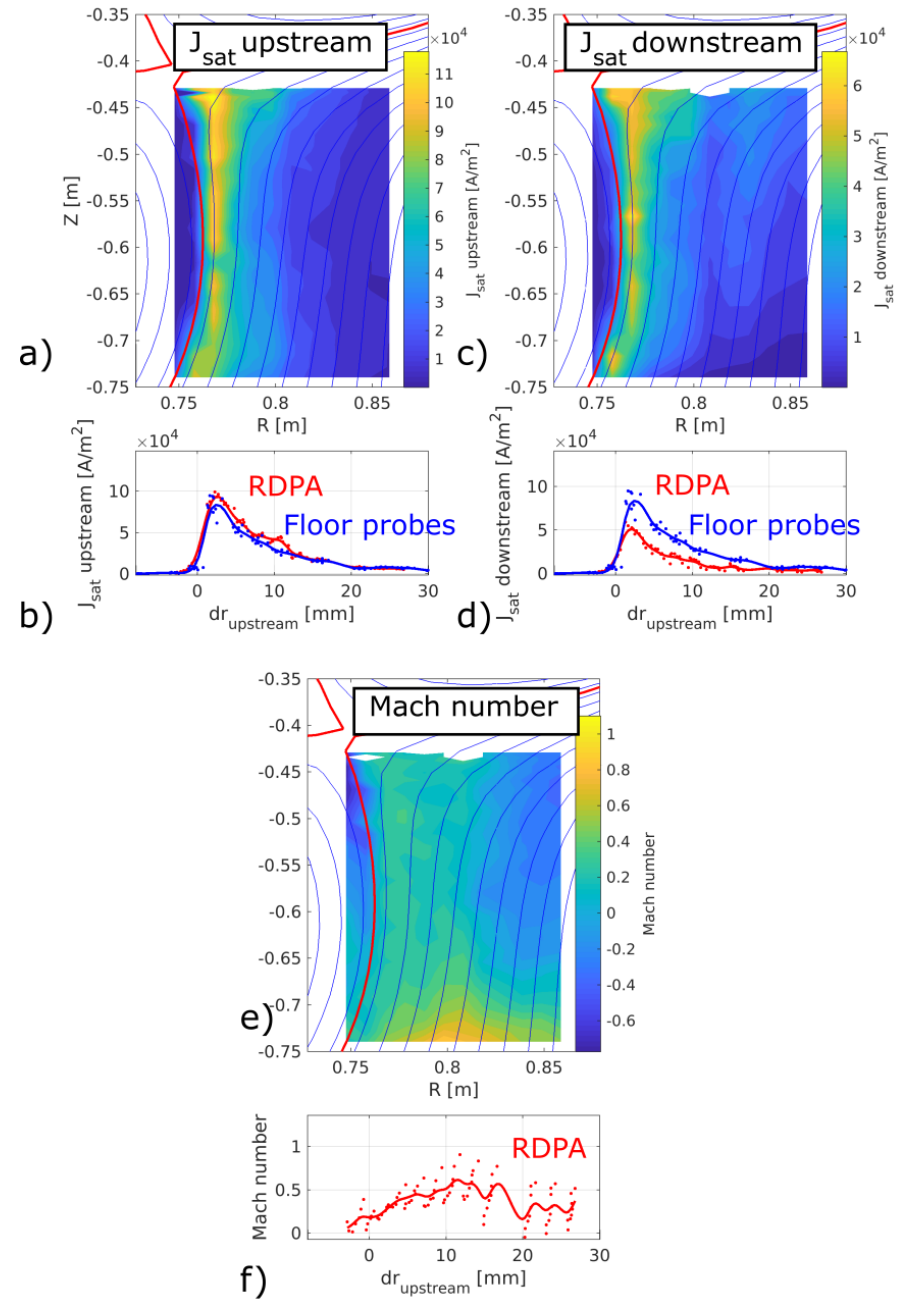

FIG. 8. a) $J_{\text {sat }}$ for the upstream RDPA probe tips in the shot \#63029 (L-mode, $I_{p}=320 k A$, unfavourable field direction for $\mathrm{H}$-mode access) and $\mathbf{b}$ ) comparison near the target with the wall-embedded probes measurements. c) and d) $J_{\text {sat }}$ for the downstream tips. e) Mach number computed from the ratio between upstream and downstream $J_{\text {sat }}$ and f) Mach number values near the target.

presented. This data is shown using a colour map, see 7 a) and c) (linear interpolation of contour lines between the measurement points shown in FIG. 7 b). One of the most straightforward plasma quantity that can be derived from LPs is $J_{\text {sat }}$, shown for the upstream probe tips in FIG. 7 a) and c). The upstream $J_{\text {sat }}$ is usually in agreement with the wall-embedded LPs profile, see FIG. 8 b). The downstream $J_{\text {sat }}$ is usually lower than the upstream $J_{\text {sat }}$, see FIG. 8 d), indicating a net ion flow towards the target. The ratio between the upstream and downstream $J_{\text {sat }}$ enables to calculate the Mach number, see FIG. 8 e) and f), with the expression $4 M=\ln \left(\frac{J_{\text {sat, upstream }}}{J_{\text {sat,downstream }}}\right) / 2.2$. In general, moderate Mach numbers are measured $M<0.8$. Negative Mach 

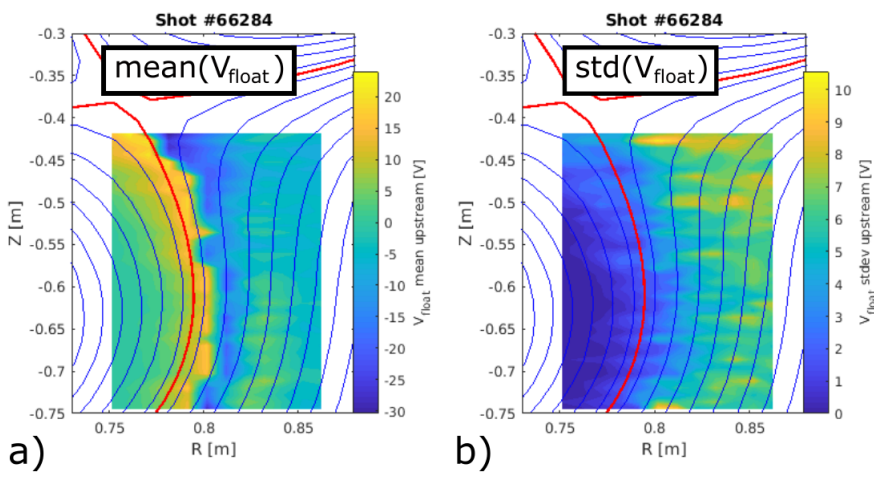

FIG. 9. a) Time averaged $V_{f l}$ measured by the upstream tips of RDPA for the shot \#66284 (L-mode, $I_{p}=160 \mathrm{kA}$, $\left\langle n_{e}\right\rangle / n_{g r}=0.27$, favourable field direction for H-mode access) and b) standard deviation of $V_{f l}$ for the same case.

numbers have also been measured locally $-0.4<M<0$, corresponding to a parallel ion flux going away from the target, caused by either strong $E \times B$ drifts or when the measurement is performed above the ionisation front.

Similarly to $J_{\text {sat }}$, the floating potential $\left(V_{f l}\right)$, FIG. 9 a), can be measured at the acquisition sampling frequency $2 \mathrm{MHz}$. The standard deviation of the signal, see FIG. 9 b), can be used as a proxy to quantify the fluctuation level at the micro-turbulence timescale. Sweeping of the voltage enables to determine time averaged quantities such as the electron temperature $\left(T_{e}\right)$. The results have been obtained with a 4 parameters fit of the $I V$ curve ${ }^{19134}$. In the example shown in FIG. 10, the upstream and downstream $T_{e}$ profiles agree with both the Thomson scattering measurement near the X-point (except, of course, in the private flux region, $d r_{\text {upstream }}<$ 0 ), and with the LPs at the target. The difference between the upstream and downstream $T_{e}$ is relatively small, which is necessary for the validity of the Mach probe model ${ }^{4}$ used to interpret plasma flows. Another quantity that can be obtained with the diagnostic is the plasma potential $\left(V_{p l}\right)$, calculated from sheath theory ${ }^{3} V_{p l}=\left(V_{f l, \text { downstream }}+V_{f l, \text { upstream }}\right) / 2+3 T_{\text {e, upstream }}$ where $T_{e, \text { upstream }} \approx T_{e, \text { downstream }}$ is, again, expressed in $\mathrm{eV}$. The average between the upstream and downstream $V_{f l}$ is taken for the $V_{f l}$ value by default, and yielded a reasonable quantitative agreement with the $V_{p l}$ target profiles, see FIG. 10 c). The derivation of a theoretical model would be required to interpret the asymmetries often seen between the downstream and upstream $V_{f l}$, suspected to be caused by electrical currents.

\section{Conclusion}

A novel fast probe array has been built at TCV, providing unprecedented $2 D$ Langmuir probe measurements across the divertor of quantities temporally resolved at the turbulence time scales (e.g. $J_{s a t}, M$ and $V_{f l}$ ) and time averaged quantities (e.g. $\left.T_{e}, n_{e}, V_{p l}\right)$. The engineering design
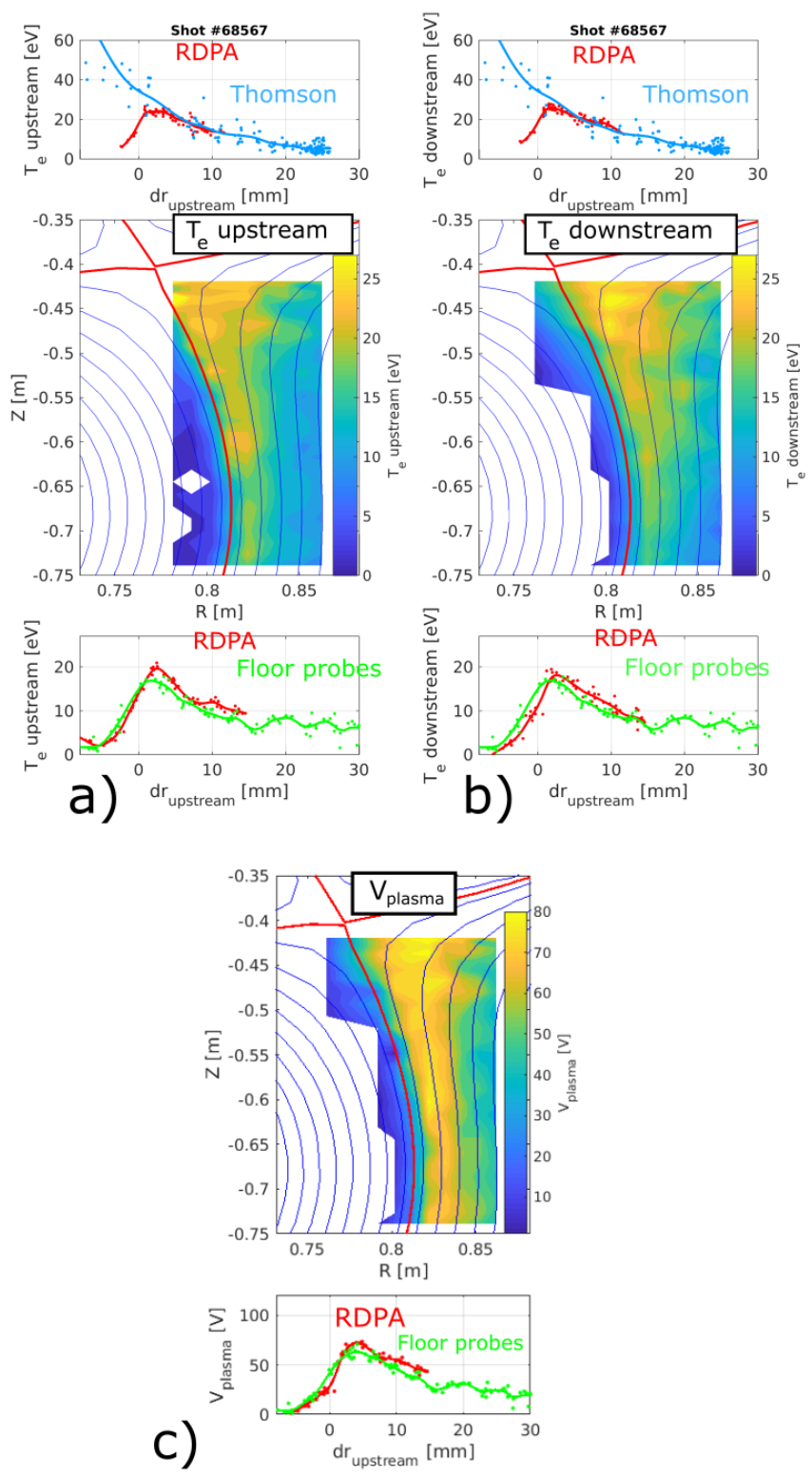

FIG. 10. a) Electron temperature measurement from the upstream RDPA probe tips for the shot \#68567 (L-mode, $I_{p}=160 \mathrm{kA},\left\langle n_{e}\right\rangle / n_{g r}=0.26$, unfavourable field direction for H-mode access) compared with Thomson scattering near the X-point and with LPs near the target, b) same results for the downstream RDPA probe tips and c) $V_{p l}$ profile compared with wall-embedded probes near the target.

answered the main challenges posed by the harsh tokamak environment. No sign of damage could be seen on the probe after more than 200 experiments performed, including $\approx 20$ disruptions. The measurements are consistent with other reference diagnostics (upstream electron Thomson scattering and downstream wall embedded LPs) for most plasma conditions, and open up vast new capabilities in terms of experimental divertor studies on TCV and validation of edge transport and turbulence 
codes. Further future work has been identified such as improving the transmission line assembly and developing a model for the interpretation of the asymmetries between upstream and downstream floating potentials.

\section{Acknowledgments}

The authors would like to particularly thank Richard Pitts (ITER), Dan Brunner (MIT), Brian LaBombard (MIT), Theodore Golfinopoulos (MIT), René Chavan (SPC) and Cedric Tsui (SPC) for their valuable advice that helped throughout the development process of the diagnostic. This work has been carried out within the framework of the EUROfusion Consortium and has received funding from the Euratom research and training program 2014 - 2018 and 2019 - 2020 under grant agreement No 633053. The views and opinions expressed herein do not necessarily reflect those of the European Commission. This work was supported in part by the Swiss National Science Foundation.

\section{Data availability}

The data that supports the findings of this study are available within the article.

\section{References}

${ }^{1}$ S. Coda and al, Nuclear Fusion 59, 112023 (2019)

${ }^{2}$ H. M. Mott-Smith and I. Langmuir, Physical Review 28, 727 (1926)

${ }^{3}$ P. C. Stangeby, The Plasma Boundary of Magnetic Fusion Devices, Series in Plasma Physics and Fluid Dynamics (Taylor and Francis, 2000).

${ }^{4}$ I. H. Hutchinson, Principles of Plasma Diagnostics (Cambridge University Press, 2005).

${ }^{5}$ H. De Oliveira, P. Marmillod, C. Theiler, R. Chavan, O. Février, B. Labit, P. Lavanchy, B. Marlétaz, R. A. Pitts, and TCV Team, Review of Scientific Instruments 90, 083502 (2019)

${ }^{6}$ J. A. Boedo, N. Crocker, L. Chousal, R. Hernandez, J. Chalfant, H. Kugel, P. Roney, J. Wertenbaker, and NSTX Team, Review of Scientific Instruments 80, 123506-123506-10 (2009)

${ }^{7}$ C. K. Tsui, J. A. Boedo, J. R. Myra, B. Duval, B. Labit, C. Theiler, N. Vianello, W. A. J. Vijvers, H. Reimerdes, S. Coda, O. Février, J. R. Harrison, J. Horacek, B. Lipschultz, R. Maurizio, F. Nespoli, U. Sheikh, K. Verhaegh, N. Walkden, and E. M. T. TCV Team, Physics of Plasmas 25, 072506 (2018)

${ }^{8}$ A. Perek, W. A. J. Vijvers, Y. Andrebe, I. G. J. Classen, B. P. Duval, C. Galperti, J. R. Harrison, B. L. Linehan, T. Ravensbergen, K. Verhaegh, M. R. de Baar, and E. M. T. TCV Team, Review of Scientific Instruments 90, 123514 (2019).

${ }^{9}$ H. Arnichand, Y. Andrebe, P. Blanchard, S. Antonioni, S. Couturier, J. Decker, B. P. Duval, F. Felici, C. Galperti, P. F. Isoz, P. Lavanchy, X. Llobet, B. Marlétaz, P. Marmillod, and J. Masur, Journal of Instrumentation 14, C09013 (2019)

${ }^{10}$ K. Verhaegh, B. Lipschultz, B. P. Duval, A. Fil, M. Wensing, C. Bowman, and D. S. Gahle, Plasma Physics and Controlled Fusion 61, 125018 (2019), 1903.08157 [physics.plasm-ph].

${ }^{11}$ J. G. Watkins, J. Hunter, B. Tafoya, M. Ulrickson, R. D. Watson, R. A. Moyer, J. W. Cuthbertson, G. Gunner, R. Lehmer,
P. Luong, D. N. Hill, M. Mascaro, J. I. Robinson, R. Snider, and R. Stambaugh, Review of Scientific Instruments 68, 373 (1997).

${ }^{12}$ M. Tsalas, N. Tsois, V. Rohde, J. Neuhauser, and ASDEX Upgrade Team, Journal of Nuclear Materials 337, 751 (2005).

${ }^{13}$ C. S. Pitcher, H. S. Bosch, A. Carlson, C. Dorn, A. Field, A. Herrmann, J. Neuhauser, T. Richter, and W. Schneider, "First results with the in-vessel probe on ASDEX-Upgrade," (1993).

${ }^{14}$ N. Asakura, S. Sakurai, N. Hosogane, M. Shimada, K. Itami, Y. Koide, and O. Naito, Nuclear Fusion 39, 1983 (1999).

${ }^{15}$ J. A. Boedo, R. Lehmer, R. A. Moyer, J. G. Watkins, G. D. Porter, T. E. Evans, A. W. Leonard, and M. J. Schaffer, Journal of Nuclear Materials 266, 783 (1999)

${ }^{10}$ J. A. Boedo, M. J. Schaffer, R. Maingi, and C. J. Lasnier, Physics of Plasmas 7, 1075 (2000)

${ }^{1}$ S. J. Zweben, Physics of Fluids 28, 974 (1985)

${ }^{18}$ M. Hubeny, D. Höschen, M. Rack, O. Neubauer, S. Bozhenkov, G. Czymek, B. Unterberg, R. König, D. Hathiramani, S. Brezinsek, and C. Linsmeier, Nuclear Materials and Energy 18, 77 (2019)

${ }^{19}$ O. Février, C. Theiler, H. De Oliveira, B. Labit, N. Fedorczak, and A. Baillod, Review of Scientific Instruments 89, 053502 (2018)

${ }^{20}$ C. S. MacLatchy, C. Boucher, D. A. Poirier, and J. Gunn, Review of Scientific Instruments 63, 3923 (1992)

${ }^{21}$ J. P. Gunn, C. Boucher, P. Devynck, I. Duran, K. Dyabilin, J. Horaček, M. Hron, J. Stöckel, G. van Oost, H. van Goubergen, and F. Žáček, Physics of Plasmas 8, 1995 (2001)

${ }^{22}$ C. K. Tsui, D. A. Taussig, M. G. Watkins, R. L. Boivin, and P. C. Stangeby, Review of Scientific Instruments 83, 10D723 (2012)

${ }^{23}$ A. Gallo, N. Fedorczak, R. Maurizio, C. Theiler, S. Elmore, B. Labit, H. Reimerdes, F. Nespoli, P. Ghendrih, and T. Eich, Nuclear Materials And Energy 12, 6. 893 (2017)

${ }^{24}$ R. Maurizio, B. Duval, B. Labit, H. Reimerdes, M. Faitsch, M. Komm, U. Sheikh, C. Theiler, and the TCV team and, Nuclear Fusion 61, 024003 (2021)

${ }^{25}$ C. Theiler, B. Lipschultz, J. Harrison, B. Labit, H. Reimerdes, C. Tsui, W. A. J. Vijvers, J. A. Boedo, B. P. Duval, S. Elmore, P. Innocente, U. Kruezi, T. Lunt, R. Maurizio, F. Nespoli, U. Sheikh, A. J. Thornton, S. H. M. van Limpt, K. Verhaegh, N. Vianello, the TCV Team, and the EUROfusion MST1 Team, Nuclear Fusion 57, 072008 (2017)

${ }^{26} \mathrm{R}$. Chavan, Internal report at SPC (1999).

${ }^{27}$ R. A. Pitts, R. Chavan, and J.-M. Moret, Nuclear Fusion 39, 1433 (1999)

${ }^{2 \gamma}$ R. Behn, A. Alfier, S. Y. Medvedev, G. Zhuang, R. Pasqualotto, P. Nielsen, Y. Martin, and TCV Team, Plasma Physics and Controlled Fusion 49, 1289 (2007).

${ }^{29}$ J. Marki, R. A. Pitts, T. Eich, A. Herrmann, J. Horacek, F. Sanchez, and G. Veres, Journal of Nuclear Materials 363, 382 (2007)

${ }^{30}$ K. Lackner, S. Guenter, P. Lauber, G. Pautasso, B. Scott, and M. Tran, Equilibrium and Macroscopic Stability of Tokamaks (International Atomic Energy Agency (IAEA), 2012) p. 348.

${ }^{31}$ E. L. Murphy and R. H. Good, Phys. Rev. 102, 1464 (1956)

${ }^{32}$ I. A. Morozov, R. A. Morozova, T. V. Dubovik, A. I. Itsenko, V. M. Panashenko, A. A. Rogozinskaya, V. S. Tsyganenko, and V. V. Lychko, in Carbon Nanomaterials in Clean Energy Hydrogen Systems (Springer Netherlands, Dordrecht, 2008) pp. 461466 .

${ }^{33}$ D. Brunner, A. Q. Kuang, B. LaBombard, and W. Burke, Review of Scientific Instruments 88, 073501 (2017)

${ }^{34}$ J. P. Gunn, C. Boucher, B. L. Stansfield, and S. Savoie, Review of Scientific Instruments 66, 154 (1995) 